\title{
Reducing inspection time of active thermographic non-destructive testing based on thermal wave theory
}

\author{
by M. Ishikawa*, M. Koyama**, H. Kasano***, R. Fukui ${ }^{\star * * *}$, \\ H. Hatta**** , S. Utsunomiya ${ }^{* * * *}$, H. Nishino* \\ * Tokushima University, Graduate School of Technology, Industrial and Social Sciences, 2-1 Minamijousanjima- \\ cho, 770-8506, Tokushima, Japan, m.ishikawa@tokushima-u.ac.jp \\ ** Meisei University, School of Science and Engineering, 2-1-1 Hodokubo, Hino, Tokyo, 191-8506, Japan \\ *** Nihon University, Department of Civil Engineering, Nakagawara, Tokusada, Tamura, Koriyama, Fukushima, \\ 963-8642, Japan \\ **** KJTD Co., Ltd., 1-9-29 Sumida, Higashiosaka-shi, Osaka, 578-0912, Japan \\ **** Japan Aerospace Exploration Agency, 3-1-1 Yoshinodai, Chuo-ku, Sagamihara, Kanagawa, 252-5210, \\ Japan
}

\begin{abstract}
According to the thermal wave theory, thermal waves with higher frequency components propagate faster. Based on this phenomenon, this study tried to reduce the inspection time of active thermographic non-destructive testing by using the thermal wave with high frequency components. Results of experiments for a concrete specimen showed that the inspection time required for detecting a subsurface defect became shorter with increasing the frequency of the input thermal wave. This result suggests that using high-frequency thermal waves is effective for reducing the inspection time of active thermographic non-destructive testing.
\end{abstract}

\section{Introduction}

Although active thermographic inspection is a convenient non-destructive testing method, it takes a long inspection time when inspecting low-thermal-conductivity materials (such as polymer materials) or detecting defects located far from an observation surface. In order to shorten the inspection time of the active thermographic method, this study focuses on the thermal wave theory. When a periodic temperature variation is generated on the surface of an object, the heat propagation speed in the object $(v)$ is obtained from one-dimensional thermal wave theory $[1,2]$ as

$$
v=\sqrt{\pi \alpha f}
$$

where $\alpha$ is thermal diffusivity of the object and $f$ is frequency of the periodic temperature change, respectively. This equation says that the thermal waves with a higher frequency component propagate faster, and means that using the high-frequency thermal waves is effective for reducing the inspection time because it propagates to the subsurface defects in a shorter time. In this study, the effect of shortening the inspection time by using the thermal waves with high frequency components was investigated through experiments for a concrete specimen.

\section{Verification of frequency dependency on heat propagation speed}

Relationship between the frequency of the thermal wave and the heat propagation speed in an object was experimentally verified.

\subsection{Experimental setup and specimen}

Figure 1 shows the experimental setup. In the experiments, a $500 \mathrm{~W}$ halogen light was used to heat a concrete specimen. The dimensional details of the specimen are given in figure 2. A thermocouple having four temperature measurement points (SE 61614, Anritsu Meter Co., Ltd.) was embedded in the specimen; the depths of the measurement points from the heated surface were $10,30,50$, and $70 \mathrm{~mm}$, respectively. The specimen was located $500 \mathrm{~mm}$ from the halogen light. The output power of the halogen light was controlled by a function generator (AFG3102, Tektronix Co., Ltd.) to excite thermal waves with an arbitrary frequency spectral peak. The output power was modulated in a sine wave shape with the frequencies of $0.001,0.00$, and $0.005 \mathrm{~Hz}$. 


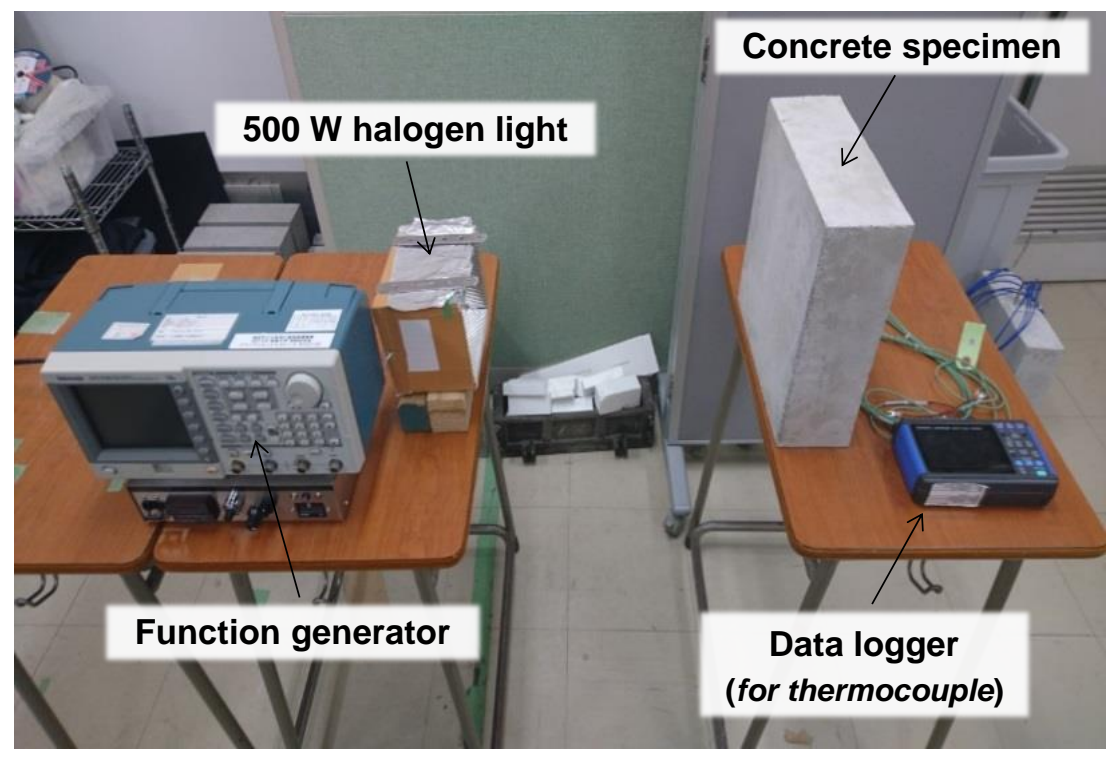

Fig. 1: Experimental setup for verification of the heat propagation speed.

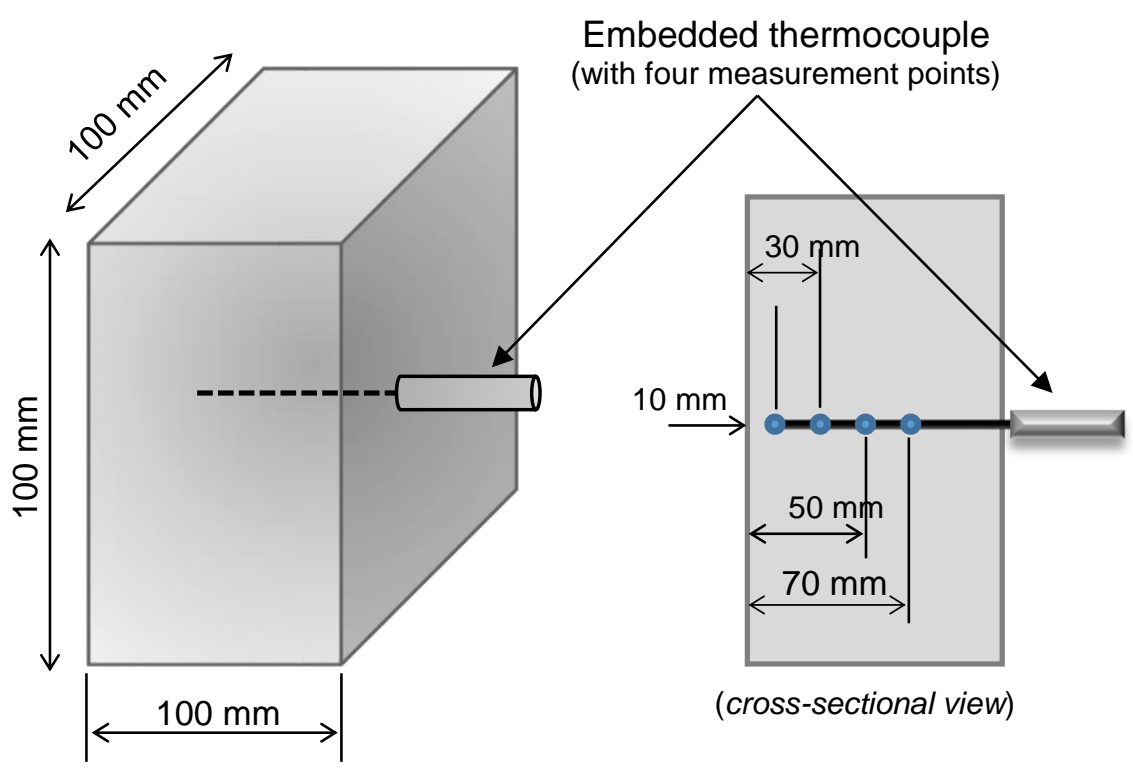

Fig. 2: Schematic diagram of a concrete specimen.

\subsection{Results}

Temperatures measured at the four measurement points when the heating frequency was $0.001 \mathrm{~Hz}$ are shown in figure 3(a). It is clearly observed that the measure temperatures are periodically increased and decreased with time, and its amplitude is decreased with the depth of measurement point. The temperatures measured at 10 and $30 \mathrm{~mm}$ depth were filtered by a bandpass filter to extract the frequency components around the excited frequency, and the filtered results are shown in figure 3(b). From the filtered temperature data, time delay between the two temperature data is obtained (see figure $3(b)$ ). This time delay means the time required to propagate the interval between the two measurement points $(20$ $\mathrm{mm}$ ), and thus the heat propagation speed is calculated from the time. Figure 4 shows the calculated heat propagation speed for each frequency condition. In figure 4, the heat propagation speed estimated from Eq. (1) (when $\alpha=0.4 \mathrm{~mm}^{2} / \mathrm{s}$ ) is also presented as a solid line. As seen in this figure, the experimentally obtained heat propagation speed increases with increasing the heating frequency, and those values well agree with the theoretical estimation. Therefore, these results verify that the thermal waves with a higher frequency component propagate faster. 
(a)

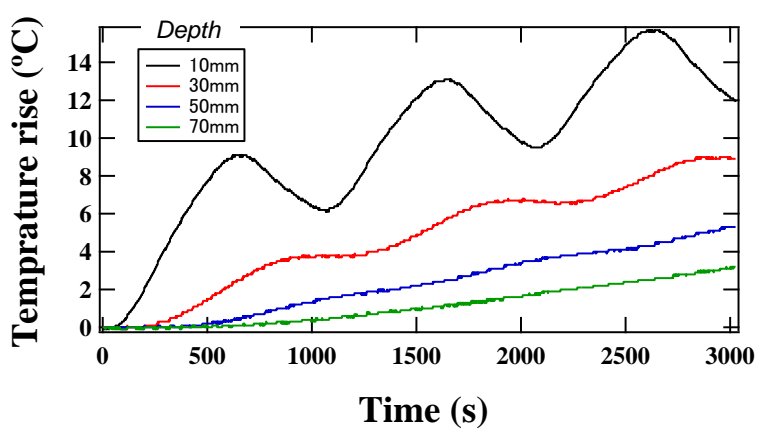

(b)

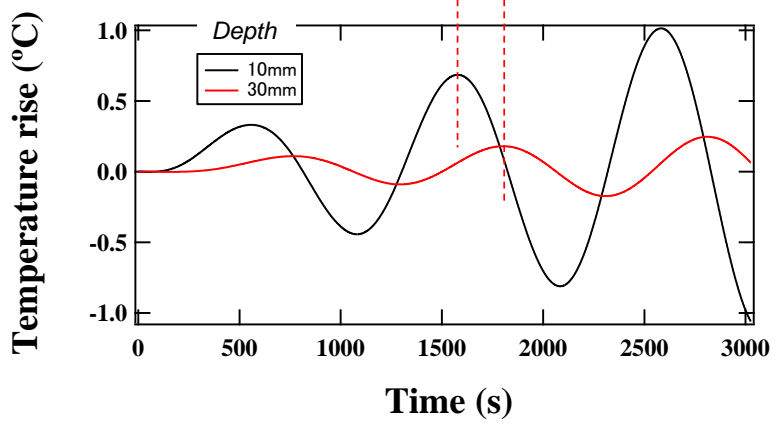

Fig. 3: (a) Temperatures measured at four measurement points in the specimen (heating frequency $=0.001 \mathrm{~Hz}$ ), (b) temperature data after applying a bandpass filter.

(a)

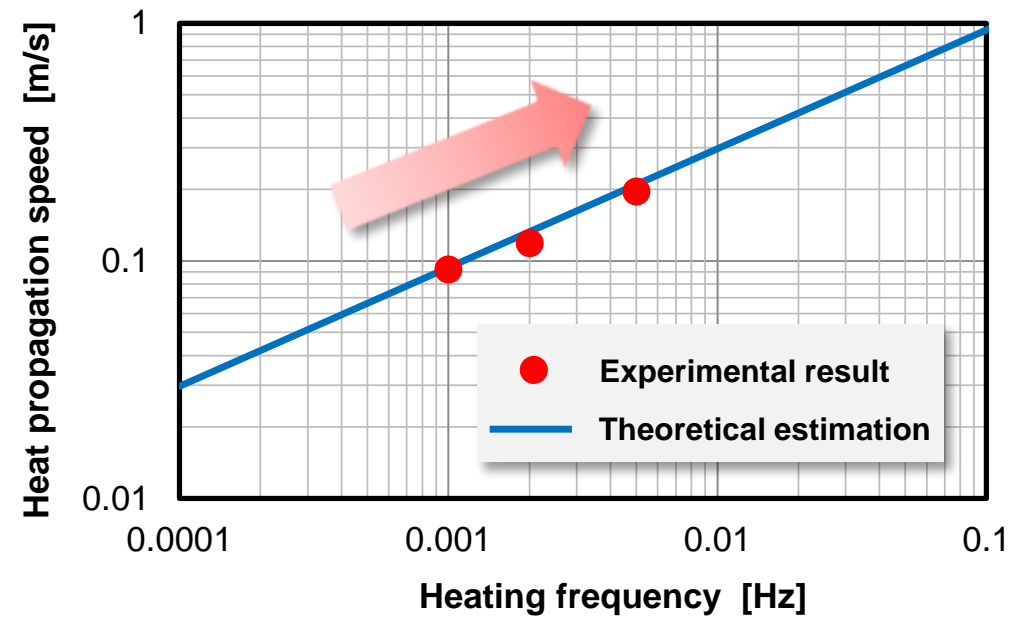

Fig. 4: Phase images obtained from temperature data for (a) $53 \mathrm{~s}$, (b) $73 \mathrm{~s}$, and (c) $93 \mathrm{~s}$.

\section{Investigation of inspection time required to detect a defect in concrete}

\subsection{Experimental setup and specimen}

Figure 5 shows the experimental setup and a concrete specimen used in the experiments. The specimen has an artificial defect (2-mm-thick air-gap with width of $100 \mathrm{~mm}$ ) at a depth of $10 \mathrm{~mm}$ from the heated surface. The specimen was periodically heated using a halogen lamp located $5 \mathrm{~m}$ away. Similar to the experiments in section 2 , the output power of the halogen lamp was controlled by a function generator into a sine wave shape (the maximum heat input was $2 \mathrm{~kW} / \mathrm{m}^{2}$ ). The heating frequency was varied as $0.01,0.03,0.05$, and $0.1 \mathrm{~Hz}$. Temperature on the heated surface during heating was monitored using an infrared camera (A315, FLIR Systems Inc.). Then, Fourier transform was applied to the observed temperature-time data to obtain phase data $[2,3,4]$. Because the phase data enables to detect a minute temperature difference caused by the defect more easily [5,6], using phase data is effective to detect the temperature change in an early stage. Thus, by using the phase data, relationship between the minimum time required to detect the defect and the frequency of the input thermal wave was examined; phase data were obtained while varying the Fourier transformed time range of the temperature data, and the minimum inspection time was determined as the time when signal to noise ratio $(\mathrm{S} / \mathrm{N})$ in the obtained phase image reaches 1.5. In addition, the same examination using a $10 \mathrm{~kJ}$ xenon flash lamp was performed for comparison (by using the instantaneous flash heating, thermal waves with broadband frequency components are simultaneously excited). 


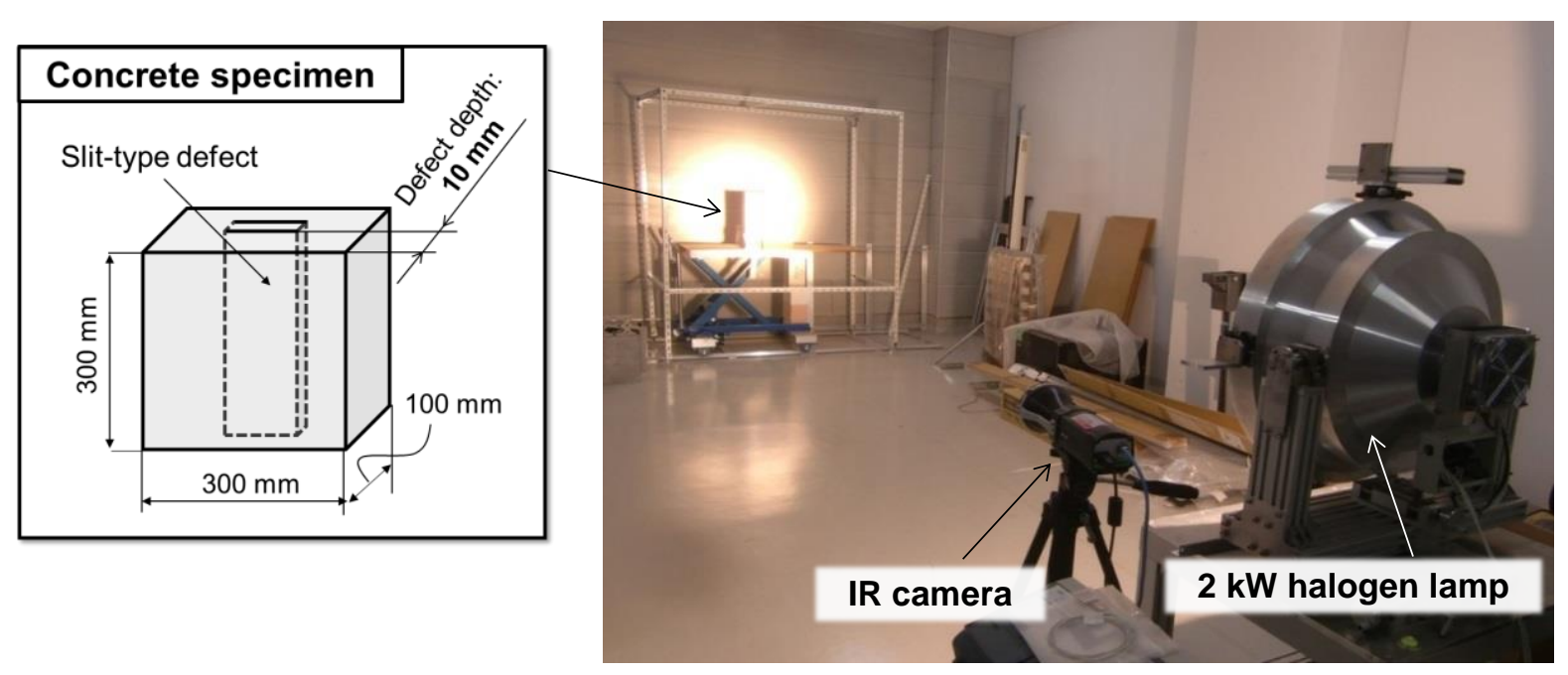

Fig. 5: Experimental setup for investigation of the inspection time required to detect a defect in a concrete.

\subsection{Results}

Figure 6 shows phase images obtained when heating frequency is $0.01 \mathrm{~Hz}$; the phase images were obtained from temperature data for 53,73 , and $93 \mathrm{~s}$. As you can see in this figure, the defective region can be detected more clearly in the phase image obtained from longer-duration temperature data. The minimum time required to detect the defect (when $\mathrm{S} / \mathrm{N}=1.5$ ) was given as $73 \mathrm{~s}$ (figure $6(\mathrm{~b})$ ). Experimentally obtained minimum inspection time for each heating frequency condition is shown in figure 7 . In the same figure, theoretically estimated minimum inspection time calculated using Eq. (1) (calculated as the round-trip propagation time between the heated surface and defect when $\alpha=0.4 \mathrm{~mm}^{2} / \mathrm{s}$ ) are also presented. It is found that the required inspection time is decreased with increasing the heating frequency. These results shows that using high-frequency periodic heating (i.e., using thermal wave with high frequency components) is effective in reducing inspection time.

Figure 8 shows phase images obtained from experiments using the xenon flash lamp. The minimum time required to detect the defect (when $\mathrm{S} / \mathrm{N}=1.5$ ) is given as $35 \mathrm{~s}$ (figure $8(\mathrm{~b})$ ); this time is shorter than that obtained when using 0.1 $\mathrm{Hz}$ periodic heating (see in figure 7). This should be because that the heat input by the instantaneous flash lamp includes thermal waves with higher frequency components than $0.1 \mathrm{~Hz}$, and the higher frequency thermal waves propagates faster. This result suggests that the inspection time will be further shorter by using thermal waves with higher frequency components.

(a)

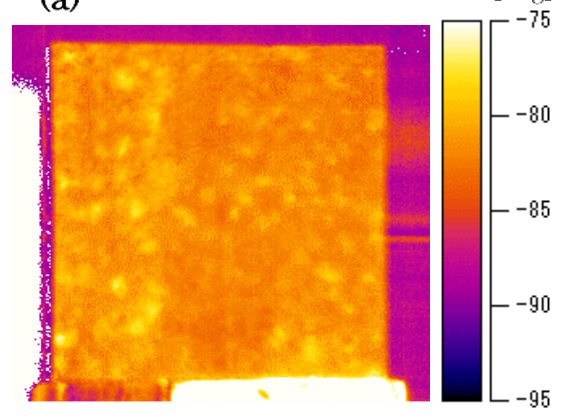

(b)

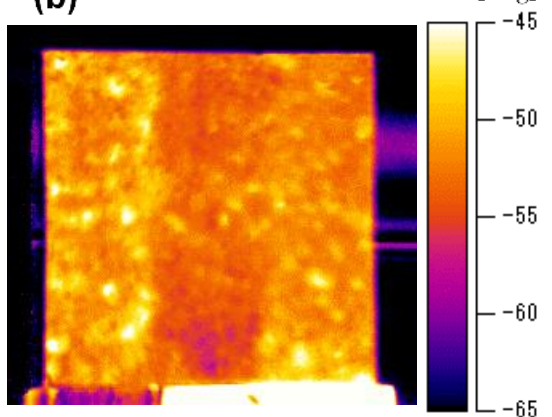

(c)

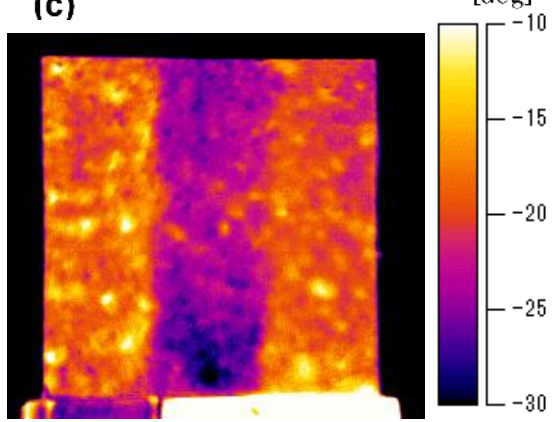

Fig. 6: Phase images obtained from temperature data for (a) $53 \mathrm{~s}$, (b) $73 \mathrm{~s}$, and (c) $93 \mathrm{~s}$. 


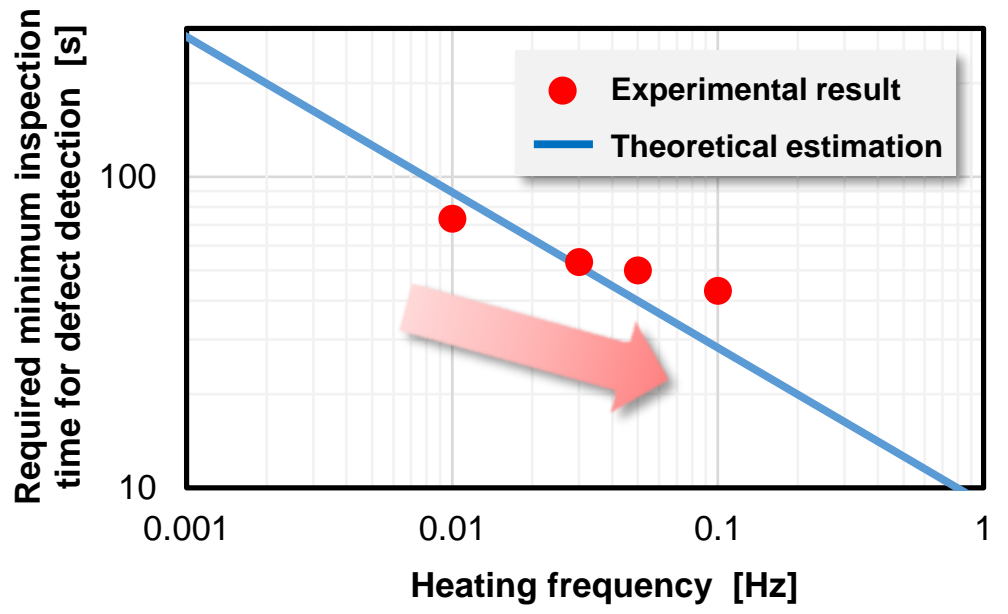

Fig. 7: Experimentally obtained relationship between the time required for detecting 10-mm depth defect and heating frequency (theoretical estimation calculated using Eq. (1) is presented as a solid line for comparison).

(a)

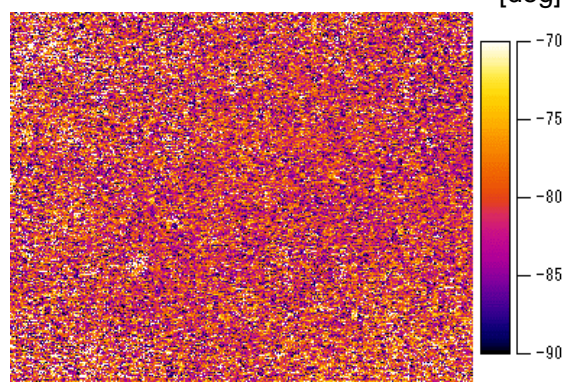

(b)

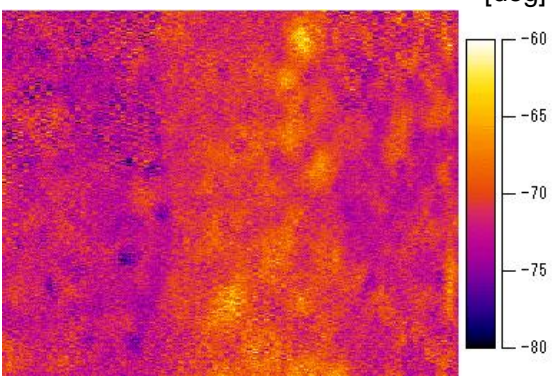

(c)

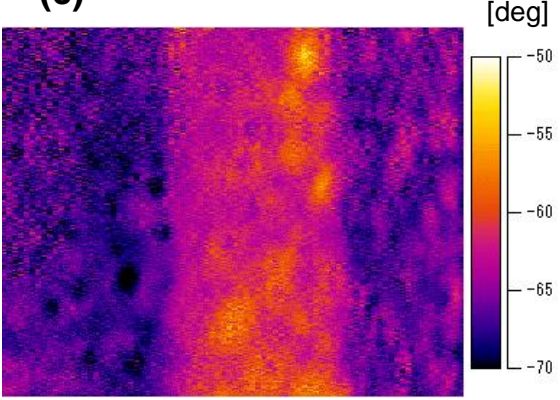

Fig. 8: Phase images obtained from an experiment by using an xenon flash lamp (displaying only the specimen area). The phase images are obtained from temperature data for (a) $25 \mathrm{~s}$, (b) $35 \mathrm{~s}$, and (c) $55 \mathrm{~s}$.

\section{Conclusion}

Reduction of inspection time of active thermography for detecting a subsurface defect was observed through experiments. Experimental results showed that: 1) heat propagation speed was increased with increasing the heating frequency, and 2) the minimum required inspection time became shorter when the thermal waves with higher frequency components were used. There results implies that the inspection in a further shorter time could be achieved by using the further high-frequency thermal waves. However, it must be noted that thermal waves are attenuated during propagation, and the attenuation ratio drastically increases with the frequency of the thermal wave. For example, when thermal wave with the frequency of $0.01 \mathrm{~Hz}$ propagates for $10 \mathrm{~mm}$ in a concrete $\left(\alpha=0.4 \mathrm{~mm}^{2} / \mathrm{s}\right)$, its amplitude is reduced to $16 \%$; when the frequency is $0.1 \mathrm{~Hz}$, the amplitude is reduced to $0.3 \%$. This reduction of the thermal wave amplitude leads to reduction of $\mathrm{S} / \mathrm{N}$ in the obtained imaged. Therefore, the effect of shorting the inspection time should be limited depending not only on the frequency of input thermal wave but also on the input heat energy, and appropriate heat input conditions must be considered.

\section{Acknowledgement}

This work was supported by JSPS KAKENHI Grant Number 17K01296. 


\section{REFERENCES}

[1] Almond DP, Patel PM. Photothermal science and techniques. London: Chapman \& Hall; 1996.

[2] Maldague $X$. Theory and practice of infrared technology for nondestructive testing. New York: John Wiley \& Sons; 2001.

[3] Maldague X, Marinetti S. Pulse phase infrared thermography, J. appl. Phys. 1996;79:2694-2698

[4] Maldague X, Galmiche F, Ziadi A. Advances in pulsed phase thermography, Infrared Phys. Technol. 2002; 43: $175-181$.

[5] Weritz F, Arndt R, Röllig M, Maierhofer C, Wiggenhauser H. Investigation of concrete structures with pulse phase thermography, Materials and Structures. 2005; 38: 843-849.

[6] Ishikawa M, Hatta H, Habuka Y, Fukui R, Utsunomiya S. Detecting deeper defects using pulse phase thermography. Infrared Phys. Technol. 2013; 57: 42-49. 